\title{
Rendición de cuentas de los medios en la era digital: visiones de los periodistas y ciudadanos catalanes y propuestas de actuación
}

\author{
Marta Narberhaus \\ Universitat Internacional de Catalunya \\ mnarberhaus@uic.es \\ https://orcid.org/0000-0002-8893-833X
}

\author{
Xavier Ramon-Vegas \\ Universitat Pompeu Fabra \\ xavier.ramon@upf.edu \\ https://orcid.org/0000-0002-4478-5626
}

\author{
Cristina Perales-García \\ Universitat Pompeu Fabra \\ cristina.perales@upf.edu \\ https://orcid.org/0000-0003-2937-2468
}

\section{Media accountability in the digital age: Catalan journalists and citizens' viewpoints and action proposals}

RESUMEN

En un escenario complejo y marcado por grandes transformaciones, la rendición de cuentas de los medios (media accountability) adquiere valor fundamental para recuperar la confianza de la opinión pública respecto al periodismo y a sus profesionales. Mediante la triangulación de métodos cuantitativos y cualitativos, esta investigación ahonda en la rendición de cuentas en el escenario catalán. Concretamente, se examina: (1) la percepción de los profesionales y los ciudadanos sobre los supuestos que afectan a la situación del periodismo en Cataluña; (2) su valoración sobre la efectividad de los instrumentos de accountability impulsados de forma interna y externa a los medios; (3) su opinión de la contribución

de los usuarios en la producción, corrección y crítica informativa; y (4) su valoración sobre los agentes ante quienes los periodistas deben sentirse responsables. Los resultados revelan que los profesionales y ciudadanos perciben de forma pesimista la situación del periodismo en Cataluña. Ambos colectivos ven como necesarios los instrumentos de rendición de cuentas, aunque existe un desconocimiento generalizado de ciertos mecanismos innovadores de participación destinados a mejorar la calidad periodística. A la luz de estos hallazgos, el trabajo formula propuestas de actuación dirigidas a medios y otros actores relevantes y aporta indicaciones para futuros estudios.

PALABRAS CLAVE

Rendición de cuentas; Medios; Periodismo; Ciudadanía; Ética periodística; Cataluña.
ABSTRACT

In a complex scenario characterized by major transformations, media accountability acquires a fundamental value to regain citizens' trust in journalism and its professionals. Through the triangulation of quantitative and qualitative methods, this research delves into accountability in the Catalan landscape. Specifically, it examines: (1) the perception of professionals and citizens on the elements that affect the situation of journalism in Catalonia; (2) their assessment of the effectiveness of the accountability instruments created within and outside media organizations; (3) their opinion of the users' contribution in the production, correction and criticism of news; and (4) their assessment of the agents to whom journalists should feel responsible. Results reveal that professionals and citizens perceive the situation of journalism in Catalonia in a pessimistic way. Both groups see accountability instruments as necessary, although there is widespread lack of knowledge of certain innovative participation mechanisms aimed at improving journalistic quality. In light of these findings, the article formulates action proposals aimed at the media and other relevant actors and provides indications for future studies.

\section{KEYWORDS}

Accountability; Media; Journalism; Citizenship; Journalism ethics; Catalonia 


\section{Introducción}

En el escenario actual, el periodismo atraviesa un período de grandes transformaciones, caracterizado por retos de primer orden que inciden sobre todas las fases del proceso productivo. Entre ellos, cabe destacar la híper-aceleración de los ciclos informativos, la creciente concentración de medios, el descenso de la inversión publicitaria, la multiplicación de canales de difusión y la progresiva expansión de prácticas vinculadas a la comercialización, como la polarización de los contenidos, el clickbait o la desinformación (Broersma, 2019; Justel, Micó y Ordeix, 2018; Masip, Ruiz-Caballero y Suau, 2018; Pickard, 2020; Zelizer, 2018). Todas estas cuestiones, sumadas a la intensificación de problemas clásicos en la profesión -como las injerencias por parte de los poderes económicos y políticos (Alsius, 2010)- han revertido en una crisis de confianza y credibilidad en el periodismo (Hanitzsch, Van Dalen y Steindl, 2018). García-de-Torres y Farmer (2017, p. 155) apuntan que "la sociedad, crítica, parece poco dispuesta a confiar en los medios periodísticos, lo que hace de esta profesión un objetivo más vulnerable ante los ataques externos". En este escenario complejo, la rendición de cuentas de los medios (media accountability) adquiere valor fundamental para recuperar la confianza de la opinión pública respecto al periodismo y a sus profesionales (Bertrand, 2018).

El concepto de rendición de cuentas hace referencia al "compromiso de los medios de responder por su práctica profesional ante la sociedad" (Rojas-Torrijos y Ramon-Vegas, 2017, p. 916). Desde la óptica de la teoría normativa de los medios, los periodistas deben considerar como prioritaria la rendición de cuentas ante los ciudadanos (Christians, Rotzoll y Fackler, 1991), salvaguardando así su independencia frente a otros actores como los gobiernos, anunciantes o grupos de interés. El estudio realizado por Harro-Loit (2015) con periodistas de 14 países certifica que los profesionales también se sienten responsables frente a su conciencia, sus fuentes y los valores democráticos, expresando una baja lealtad frente a los poderes políticos, económicos y religiosos.

La rendición de cuentas debe ser fomentada tanto por las mismas empresas periodísticas como por otros actores involucrados en el proceso comunicativo -ciudadanos y organizaciones independientes- en base a tres valores fundamentales: la transparencia, la autorregulación y la participación del público (Díez-Garrido, Campos-Domínguez y Calvo, 2019; Heikkilä et al., 2012; Ramon-Vegas, Mauri-Ríos y Alcalá-Anguiano, 2016). Estas tres dimensiones se canalizan a través de un amplio abanico de instrumentos de rendición de cuentas (media accountability instruments) (Tabla 1).

Eberwein et al. (2011) clasifican los instrumentos de rendición de cuentas en mecanismos impulsados de forma interna y externa a los medios de comunicación, distinguiendo también entre aquellos instrumentos tradicionales e innovadores. En el caso de los instrumentos tradicionales, los estudios sobre los códigos éticos y los libros de estilo han predominado en los ámbitos de investigación internacional y español (Alsius, 1999; Aznar, 1999; García-Avilés, 1996; Hafez, 2002), conjuntamente con los trabajos centrados en explorar la figura del defensor del lector (Evers, 2012; Ferrucci, 2019; Maciá-Barber, 2006; Ramon, Billings y Rojas-Torrijos, 2019; van Dalen y Deuze, 2006). Las características, funciones y limitaciones de las cartas al director (Pastor, 2010; Raeymaeckers, 2005; Wahl-Jorgensen, 2002), los escritos de rectificación (Moreno-Gil, 2019), y los consejos de la información (Aznar y Serrano-Moreno, 2017; Mauri-Ríos, Rodríguez-Martínez, Figueras-Maz y Fedele, 2018; Suárez-Villegas, 2015) también han recibido una atención académica preferente.

Herrera-Damas, Maciá-Barber y Luengo-Cruz (2018, p. 214) señalan que "los instrumentos tradicionales parecen insuficientes hoy en día para enfrentarse a los nuevos retos surgidos a raíz de la llegada de Internet y, más específicamente, la explosión de las redes sociales". Paradójicamente, son precisamente estas nuevas plataformas las que han facilitado la aparición de mecanismos como los blogs editoriales, los chats y encuentros digitales con los lectores, los botones de notificación de errores, los comentarios en las noticias y en redes sociales, los observatorios de crítica mediática o los blogs de análisis impulsados por periodistas, ciudadanos y académicos (Craft, Vos y Wolfgang, 2016; García-Avilés, 2019; Karlsson, Clerwall y Nord, 2017; Mauri-Ríos y Ramon-Vegas, 2015; Pérez-Díaz, Zamora-Medina y Arroyas-Langa, 2020; Spiller, Degen, Kronewald, y Guertler, 2016).

En la era de la deliberación digital (Masip, Ruiz-Caballero y Suau, 2019; Raeijmaekers y Maeseele, 2015), los "comentarios en las webs y las redes sociales ofrecen nuevas posibilidades para promover debates espontáneos pero organizados sobre cuestiones de interés público" (Suárez-Villegas, Rodríguez-Martínez, Ramon-Vegas, 2020, p. 2), fomentando así "la autoexpresión ciudadana, la socialización democrática y el refuerzo de la cohesión social" (Valera-Ordaz, 2017, p. 1). Desde la óptica de la rendición de cuentas, estos espacios virtuales también permiten a los ciudadanos expresar sus pensamientos y opiniones sobre el contenido publicado en los medios, promoviendo de este modo un debate y reflexión crítica sobre la práctica periodística. Otros espacios innovadores como los chats o los sistemas de notificación de errores también persiguen fomentar la participación en aras de mejorar la calidad informativa. Pese a que los periodistas aún muestran una cierta reticencia a afirmar que las críticas y comentarios efectuados por los usuarios afectan decisivamente a su práctica profesional (Fengler et al., 2015), investigaciones recientes revelan que los ciudadanos valoran de forma positiva las nuevas herramientas de participación para contribuir a la mejora periodística (Eberwein, 2019; Engelke, 2019). 


\begin{tabular}{|c|c|c|c|}
\hline Tipología & Instrumento & Carácter & Dimensión \\
\hline \multirow{11}{*}{$\begin{array}{l}\text { Instrumentos } \\
\text { de } \\
\text { accountability } \\
\text { internos }\end{array}$} & Apartado del medio con información corporativa & Innovador & Transparencia \\
\hline & Contribución de los usuarios en la creación y revisión de los contenidos & Innovador & Participación \\
\hline & Botones de corrección de errores & Innovador & Participación \\
\hline & Chats y encuentros digitales con los lectores & Innovador & Participación \\
\hline & Cartas al director & Tradicional & Participación \\
\hline & $\begin{array}{l}\text { Comentarios de los usuarios en las noticias publicadas en la web del } \\
\text { medio }\end{array}$ & Innovador & Participación \\
\hline & Crítica del periodismo en las secciones o espacios en la web del medio & Innovador & Autorregulación \\
\hline & Blog editorial del medio & Innovador & Transparencia \\
\hline & Defensor del lector & Tradicional & Autorregulación \\
\hline & Blog del defensor del lector & Innovador & Autorregulación \\
\hline & Libro de estilo del medio & Tradicional & Autorregulación \\
\hline \multirow{12}{*}{$\begin{array}{l}\text { Instrumentos } \\
\text { de } \\
\text { accountability } \\
\text { externos }\end{array}$} & Análisis académico del periodismo & Tradicional & Participación \\
\hline & Crítica en las redes sociales & Innovador & Participación \\
\hline & Blogs de medios de comunicación escritos por periodistas & Innovador & Autorregulación \\
\hline & Crítica del periodismo por bloggers periodistas & Innovador & Autorregulación \\
\hline & Observatorios de crítica de medios & Innovador & Autorregulación \\
\hline & Revistas sectoriales sobre la profesión & Tradicional & Autorregulación \\
\hline & Asociaciones de espectadores & Tradicional & Participación \\
\hline & Consejos de la información / Consejo Audiovisual & Tradicional & Autorregulación \\
\hline & Sindicatos profesionales & Tradicional & Autorregulación \\
\hline & Colegios de periodistas & Tradicional & Autorregulación \\
\hline & Códigos éticos específicos o temáticos & Tradicional & Autorregulación \\
\hline & Códigos éticos profesionales generales & Tradicional & Autorregulación \\
\hline
\end{tabular}

Tabla 1. Instrumentos de rendición de cuentas impulsados de forma interna y externa a los medios, atendiendo a su carácter tradicional o innovador y a la dimensión que fomentan. Elaboración propia a partir de las clasificaciones definidas por Eberwein et al. (2011) y Ramon-Vegas y Mauri-Ríos (2020).

\section{Cataluña: un escenario fértil para el desarrollo de iniciativas de accountability}

España es uno de los países que forman parte del modelo Mediterráneo o Pluralista Polarizado descrito por Hallin y Mancini (2004) que presenta un espectro más amplio de instrumentos de rendición de cuentas impulsados de forma interna y externa a los medios (Alsius, Rodríguez-Martínez y Mauri-Ríos, 2018). El país cuenta con una "desarrollada infraestructura de autorregulación que ha evolucionado después del fin del régimen franquista, incluyendo consejos de la información nacionales y regionales y varios defensores tanto en medios escritos como audiovisuales" (Fengler et al., 2015, p. 256). Estos instrumentos de accountability tienen como función esencial contrarrestar las limitaciones y condicionantes que históricamente han caracterizado el contexto mediático español, entre las cuales cabe señalar la influencia política sobre el periodismo, la polarización de la esfera pública, las injerencias comerciales sobre los medios, la falta de transparencia, la débil profesionalización y la precariedad laboral (Campos-Domínguez; Redondo-García, 2015; Hallin y Mancini, 2004; Luengo-Cruz, Maciá-Barber y Requejo-Alemán, 2017; Masip et al., 2018). Asimismo, estos mecanismos persiguen atajar disfunciones éticas propias del entorno digital que también afectan al escenario español, entre ellas la comodificación de los contenidos, la orientación hacía las métricas y el impacto de la desinformación en línea (Ramon-Vegas y Mauri-Ríos, 2020).

Cabe señalar que el desarrollo de los instrumentos de rendición de cuentas en España no ha sido uniforme. Como apuntan Suárez-Villegas, Rodríguez-Martínez, Mauri-Ríos y López-Meri (2017, p. 321), "la cultura periodística en España no responde a un único modelo de sistema de medios (modelo mediterráneo o pluralista polarizado), como señalan Hallin y Mancini en Comparing Media Systems, sino que la diversidad de culturas periodísticas requiere matizar esta clasificación". Como anotan Almiron, Narberhaus y Mauri-Ríos (2016), Cataluña podría situarse más cerca de los modelos Atlántico Norte o Liberal o 


\section{Democrático Corporativo}

El estudio de Rodríguez-Martínez, López-Meri, Merino-Arribas y Mauri-Ríos (2017), que analiza 60 mecanismos, ilustra precisamente la desigualdad en la implantación de instrumentos de accountability creados de forma exógena a los medios en varias comunidades autónomas (Cataluña, Galicia, Comunidad de Madrid y Comunidad Valenciana). El desarrollo y consolidación de múltiples iniciativas de rendición de cuentas en Cataluña sitúan este territorio como uno de los más avanzados en el conjunto del Estado por lo que a accountability se refiere, por delante incluso de otras comunidades como el País Vasco y Andalucía (Suárez-Villegas, Zuberogoitia y Gostín, 2019).

En este territorio han florecido iniciativas clave para la profesión, tales como el primer código de ética periodística en el estado español -Declaració de principis de la professió periodística a Catalunya (Collegi de Periodistes de Catalunya, 1992)-, la puesta en marcha del primer consejo de prensa (Consell de la Informació de Catalunya, 1997) y del primer consejo del audiovisual en España (Consell de l'Audiovisual de Catalunya, 2000) y, más recientemente, la creación y consolidación del observatorio crítico Mèdia.cat (2009) impulsado por el Grupo de Periodistas Ramon Barnils. Cataluña también sobresale en el desarrollo de iniciativas como las asociaciones de espectadores, entre las que cabe destacar la Associació de Consumidors de Mitjans Audiovisuals (TAC).

En referencia a los instrumentos creados por los propios medios, organizaciones como La Vanguardia, El Periódico de Catalunya, El Punt (actualmente, El Punt-Avui) y la Corporació Catalana de Mitjans Audiovisuals (CCMA) han sido referencia en el desarrollo e implantación de libros de estilo y los defensores de la audiencia (Alsius, 1999; Maciá-Barber, 2006; Rojas-Torrijos y Ramon-Vegas, 2017). En los últimos años, medios nativos digitales catalanes como Ara o VilaWeb han apostado por implantar mecanismos de accountability que buscan fortalecer la relación con los lectores y subscriptores, como los chats en línea con los editores (García-Avilés, 2019; Ramon-Vegas y Mauri-Ríos, 2020).

Si bien estas investigaciones han mapeado la existencia de determinados instrumentos de accountability en Cataluña, resulta primordial ir más allá para ahondar en la percepción de su efectividad (Herrera-Damas, Maciá-Barber y Luengo-Cruz, 2018) por parte de varios colectivos. Dado que "la rendición de cuentas es una cuestión que preocupa tanto a los periodistas como a las audiencias" (Chaparro-Domínguez, Suárez-Villegas y Rodríguez-Martínez, 2020, p. 813), es necesario discernir la percepción de los profesionales catalanes sobre los instrumentos de rendición de cuentas, en contraste con el conocimiento y las opiniones de los ciudadanos sobre estos mecanismos. El presente estudio pretende subsanar esta carencia en la literatura académica, ahondando también en cómo los periodistas y ciudadanos catalanes radiografían la situación del periodismo en el contexto actual.

\section{Objetivos y metodología}

Este estudio tiene como objetivo esencial ahondar en la rendición de cuentas del periodismo en la era digital, examinando la percepción de los periodistas y ciudadanos catalanes sobre cuatro cuestiones clave que inciden sobre la actividad informativa. El presente trabajo busca dar respuesta a las siguientes preguntas de investigación:

- P1. Según la percepción de los profesionales y los ciudadanos catalanes, ¿cuáles son los supuestos que afectan a la situación del periodismo en Cataluña?

- P2. ¿Cuál es la percepción de los periodistas y los ciudadanos sobre la efectividad de los instrumentos de rendición de cuentas impulsados de forma interna y externa a los medios?

- P3. ¿De qué forma los periodistas y ciudadanos catalanes valoran la contribución de los usuarios en la producción, corrección y crítica informativa?

- P4. Según los profesionales y los ciudadanos catalanes, ¿ante quién se debería sentir responsable el periodista en el ejercicio de la profesión?

Para responder estas preguntas, se apostó por la triangulación de métodos (Bryman, 2016). En primer lugar, se elaboró una encuesta para estudiar la percepción de los profesionales sobre el impacto de los instrumentos de accountability. Asimismo, la encuesta persigue obtener datos empíricos sobre varios aspectos clave de la profesión (condicionantes que afectan a la práctica informativa, relación con la audiencia y responsabilidad de los periodistas ante varios agentes). Para cubrir este espectro de cuestiones, se diseñó un cuestionario conformado por 29 preguntas. Para garantizar la operatividad del mismo, se combinaron preguntas dicotómicas, de opción múltiple y en forma de escala de valoración (Wimmer y Dominick, 2011). Para este trabajo, se han seleccionado aquellas preguntas relacionadas con las condiciones del ejercicio periodístico en Cataluña, la efectividad de los instrumentos de accountability, la participación de los usuarios y la responsabilidad profesional.

Siguiendo los criterios señalados por Weischenberg, Malik y Scholl (2006, p. 227), posteriormente empleados por el proyecto europeo Media Accountability and Transparency in Europe (MediaAcT), los encuestados debían reunir las siguientes características: (1) Trabajar para un medio de comunicación periodística (quedan excluidos de esta manera los profesionales que desempeñen tareas de relaciones públicas); (2) Realizar el ejercicio periodístico (quedan excluidos los profesionales que desempeñen tareas técnicas o de organización en la industria de los medios de comunicación); y (3) Tener una 
ocupación a tiempo completo o principalmente, es decir, ganar el 50 por ciento o más de sus ingresos de su profesión como periodista.

En contraste con otros países como Alemania, Francia o Finlandia, España no dispone de un censo o directorio oficial de profesionales del periodismo (Rodríguez-Martínez, Mauri-Ríos y Fedele, 2017). Para obtener un número suficiente de respuestas, se buscó la colaboración de instituciones profesionales como la Federación de Asociaciones de Periodistas de España (FAPE) y el Col.legi de Periodistes de Catalunya. El cuestionario se administró de forma online a través de la plataforma SurveyMonkey, y se mantuvo abierta durante tres meses (17 de octubre de 2017 - 17 de enero de 2018). El total de respuestas obtenidas en el conjunto del estado español fue de 228 ( $N=228$ ). Este artículo presenta los resultados correspondientes a los periodistas que desarrollan su tarea profesional en Cataluña $(\mathrm{N}=53)$.

Los datos resultantes se triangularon posteriormente con la información obtenida de los grupos de discusión con ciudadanos (Bryman, 2016). Esta aproximación cualitativa ha revelado datos relevantes sobre la valoración de los ciudadanos sobre la situación actual del periodismo, su percepción de los mecanismos tradicionales y de más reciente implantación y su opinión de ante quién se debería sentir responsable el periodista. Entre abril y mayo de 2018 se celebraron un total de 6 grupos de discusión en diferentes ciudades españolas (Barcelona, Castelló, Madrid, Sevilla, Mondragón y Santiago de Compostela). Para reclutar a los participantes, se empleó la aproximación denominada research-driven recruitment strategy (Peek y Fothergill, 2009). A través de esta aproximación, los miembros del equipo de investigación -compuesto por 6 universidades con sede en las ciudades mencionadas- fueron responsables de seleccionar a los participantes de cada focus group.
Para garantizar la consistencia en el enfoque metodológico, el guion de los grupos focales se basó en la encuesta a periodistas, centrándose en cuatro cuestiones clave vinculadas a la actividad periodística: los supuestos que afectan al periodismo, la efectividad de los instrumentos de accountability, la contribución de los usuarios en el proceso periodístico y la responsabilidad de los profesionales ante varios agentes. Cada una de las sesiones fue grabada y transcrita íntegramente para su análisis posterior. Este artículo ahonda en los resultados clave del grupo de discusión desarrollado en Cataluña. Con el fin de garantizar la confidencialidad de los datos, las citas se presentan únicamente con el género de cada participante (H: Hombre; M: Mujer).

\section{Resultados}

\subsection{Valoración por parte de los periodistas}

\subsubsection{Supuestos que afectan a la situación del periodismo en Cataluña}

Los resultados de las encuestas realizadas a profesionales de la comunicación en Cataluña arrojan que la retribución percibida afecta de forma decisiva la situación general del periodismo en la Comunidad Autónoma (Figura 1). Este supuesto obtiene una cualificación de 8,23 en una escala de 10. Como segundo elemento que afecta al ejercicio de la profesión están las presiones económicas que los periodistas dicen recibir (un 7,80 sobre 10) y la politización de los medios de comunicación para los que trabajan $(7,89)$.

Como elementos de menos valoración, aunque con una nota considerable en la afectación del ejercicio de periodismo, está la formación reglada de estudios específicos en Periodismo o

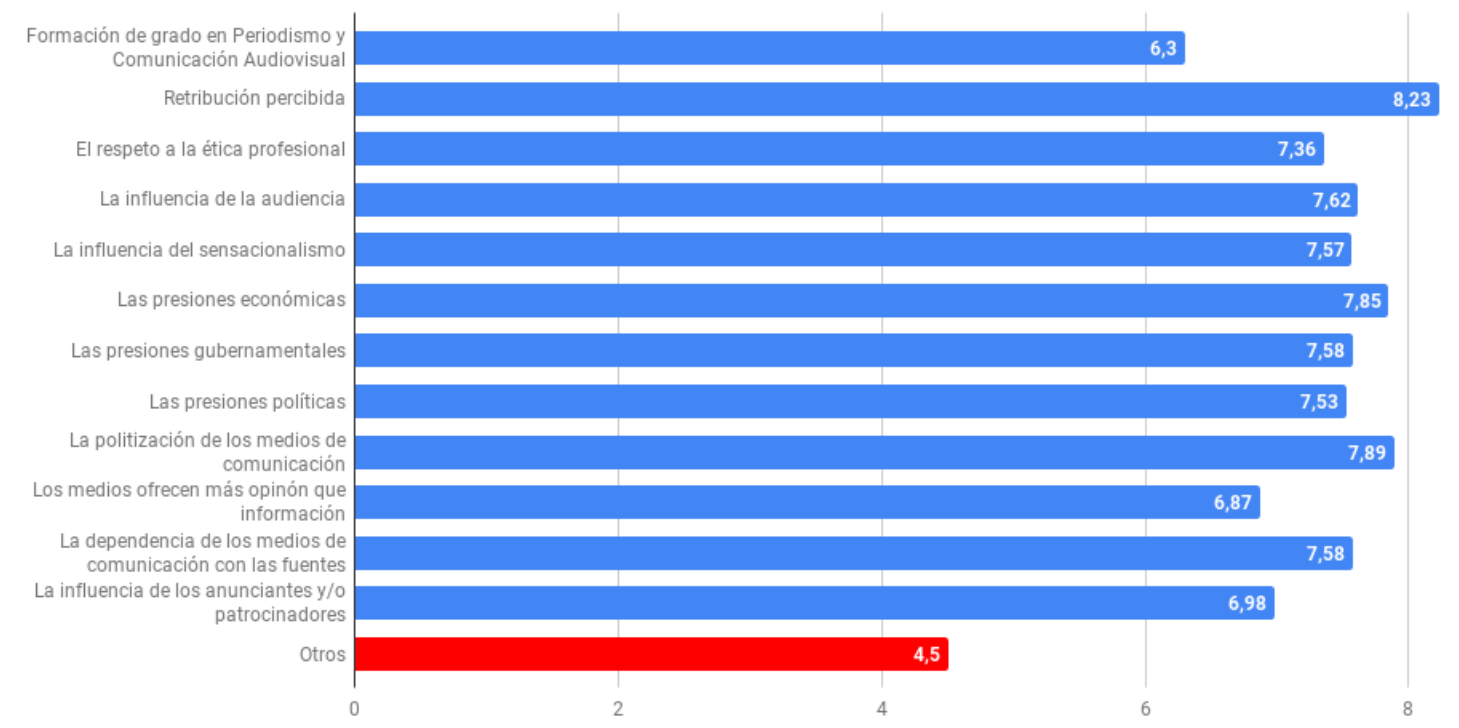

Figura 1. Afectación a la situación del periodismo en Cataluña. Elaboración propia. 


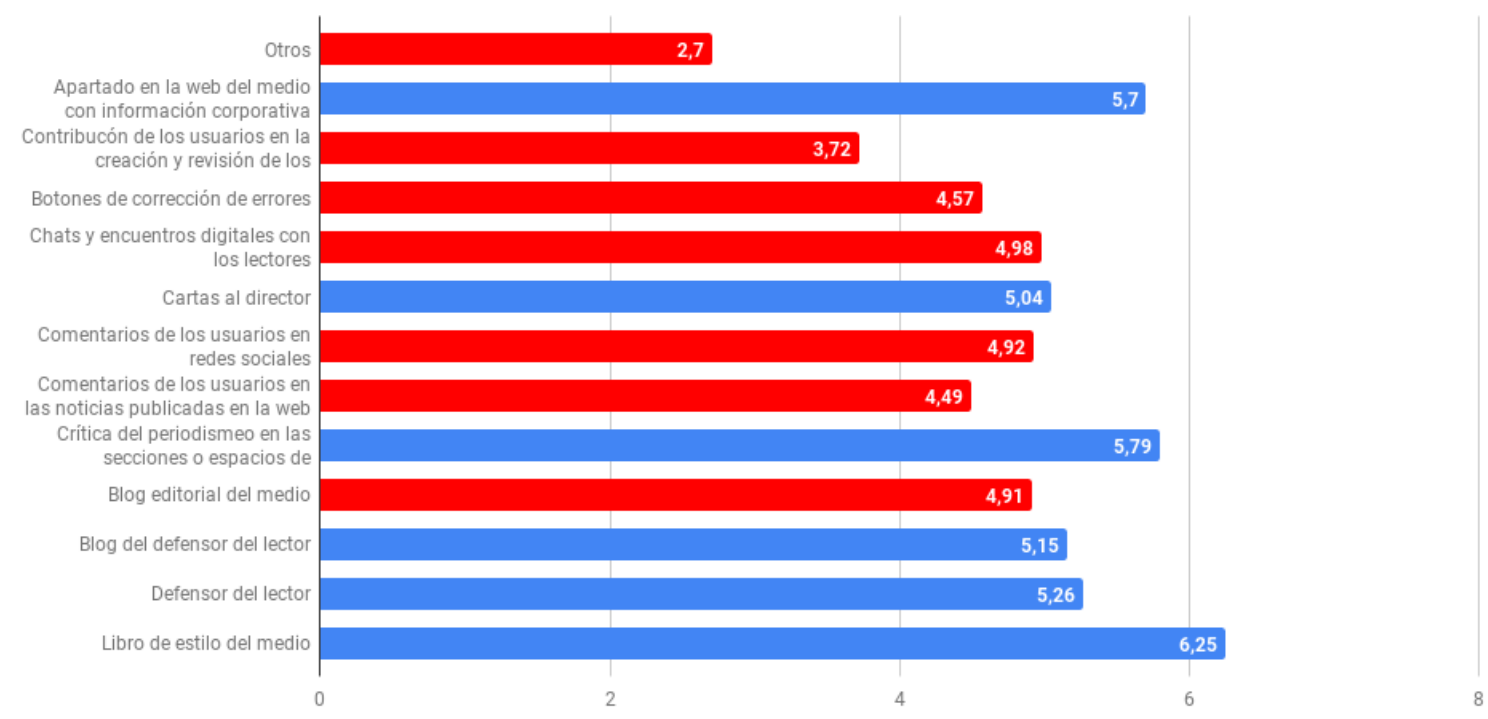

Figura 2. Valoración de los instrumentos de accountability internos. Elaboración propia.

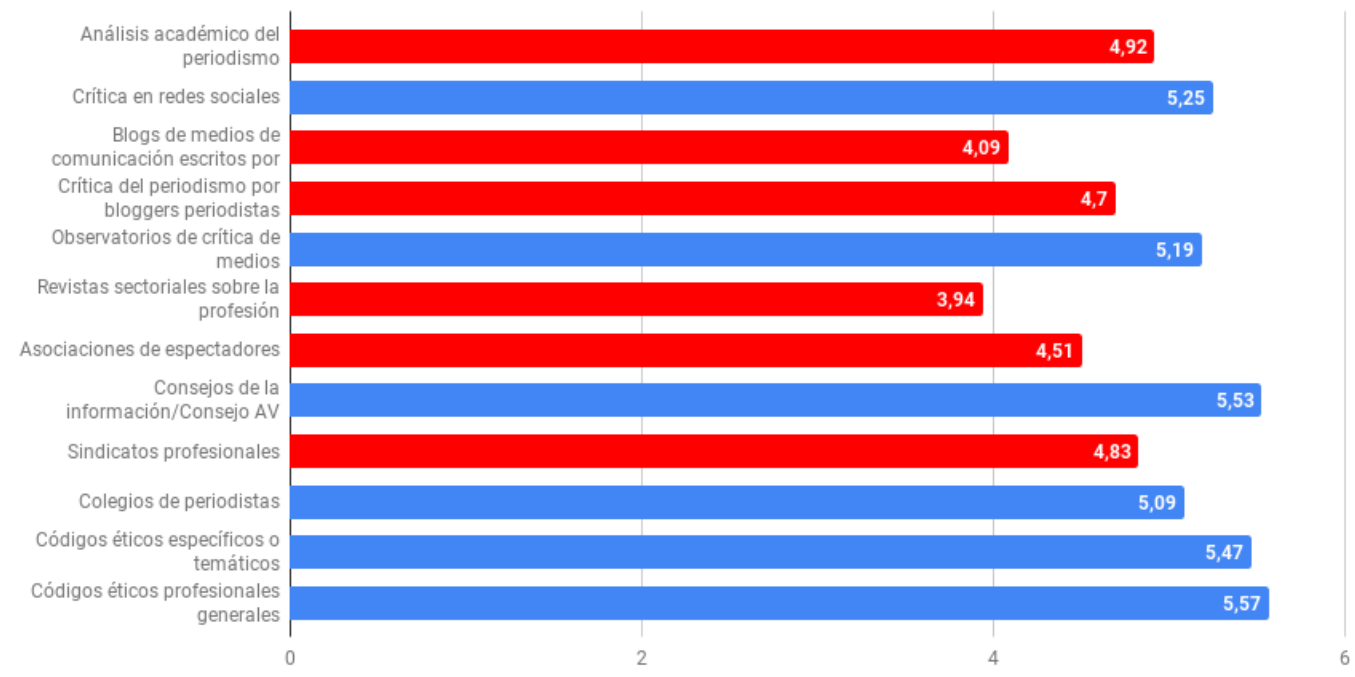

Figura 3. Valoración de los instrumentos de accountability externos. Elaboración propia.

Comunicación Audiovisual, que la valoran con un 6,3 y el hecho que los medios ofrezcan más opinión que información (6,87).

\subsubsection{Percepción de los periodistas sobre los instrumentos de accountability}

En cuanto a los instrumentos de rendición de cuentas internos con los que cuentan los periodistas para ejercer su profesión con garantías, los libros de estilo son lo que más nota reciben, aunque con un escaso 6,25 de valoración. Llama la atención (como puede verse en la Figura 2) que los mecanismos de accountability no resultan elementos legitimadores por parte de los profesionales, que suspenden la mayoría de estos instrumentos como son la contribución de los usuarios en la creación o revisión de contenidos, los chats o encuentros digitales con lectores o los comentarios de los usuarios, entre otros.

Sin embargo, si nos centramos en los mecanismos de accountability externos (Figura 3), los profesionales catalanes valoran de manera efectiva, aunque con un aprobado muy justo $(5,57)$ los códigos éticos profesionales. El Collegi de Periodistes de Catalunya recibe un 5,09 de aprobación, lo que infiere que en general no hay una legitimidad clara con los instrumentos de accountability impulsados de forma exógena a las organizaciones periodísticas. Todos los mecanismos que están relacionados con la iniciativa de los usuarios/consumidores reciben un suspenso, así como el sindicato, revistas sectoriales y los estudios académicos sobre el periodismo.

A pesar de estos bajos resultados, el 66\% de los encuestados consideran que estos mecanismos contribuyen a fomentar la responsabilidad, es decir, la calidad informativa, el pluralismo 


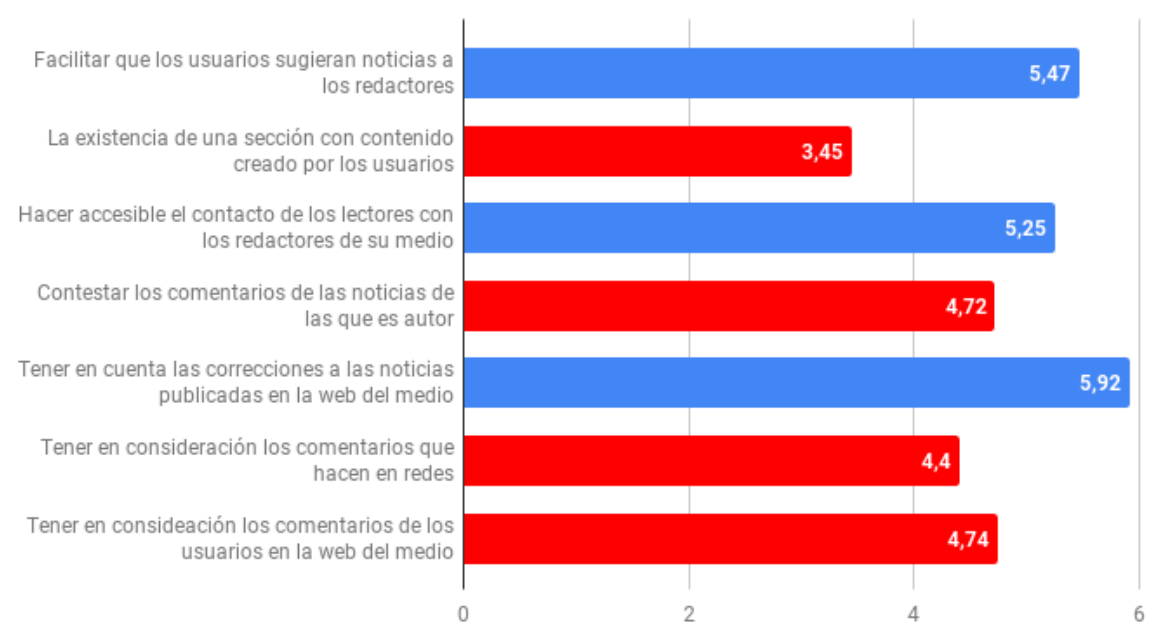

Figura 4. Valoración de la participación de los usuarios (0-10 en importancia). Elaboración propia.

y la transparencia mediática, frente a un 34\% que considera lo contrario.

\subsubsection{Los periodistas ante la contribución de los usuarios}

En referencia a las críticas del público como elemento de mejora para el buen funcionamiento periodístico, los periodistas consideran que las correcciones que el usuario pueda hacer a las noticias publicadas deben tomarse en cuenta (Figura 4), siempre que se haga a través de la página web del medio $(5,92)$, pero suspende la aportación del público en cuanto a los comentarios que puedan hacer a través de redes sociales $(4,4)$ o bien comentarios en redes (4,74). Sólo se dota con un 5,47 al hecho que el medio pueda facilitar al usuario sugerencias a los profesionales, lo que equivale a una falta de confianza en el usuario como aportador de información (ver Figura 5).

En cuanto a la interacción de los usuarios a través de las redes sociales, los periodistas catalanes la valoran con un suspenso $(4,68)$. La apreciación más alta (aunque sin llegar al aprobado) es la de considerar noticias compartidas en la cuenta de Facebook del medio de comunicación $(4,7)$.

Según los datos (ver Figura 5), los periodistas no son partidarios de apostar por un cambio en el paradigma profesional amparado en el concepto del citizen journalism, aunque se considere que es importante proporcionar un espacio de intercambio con los usuarios.

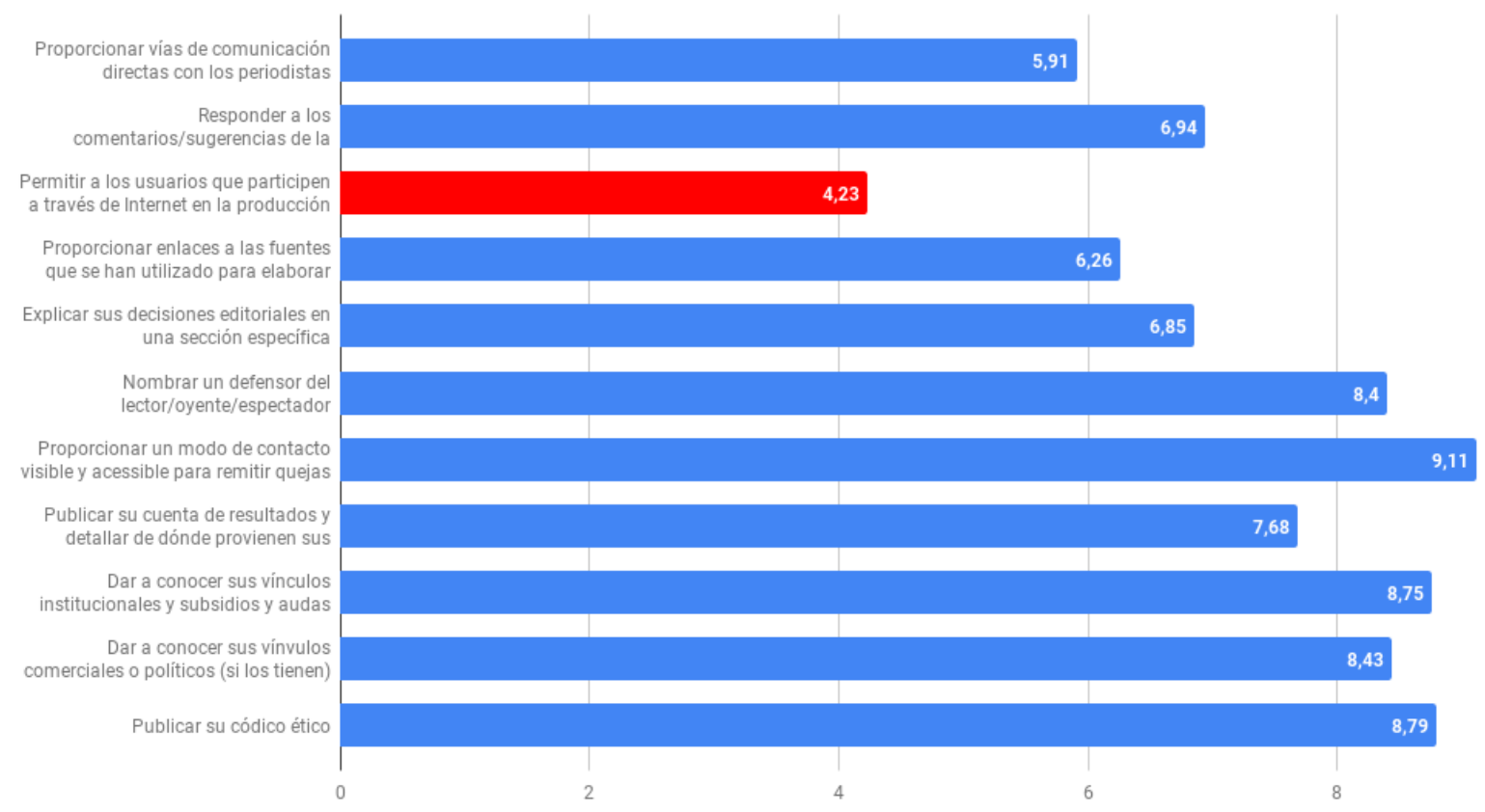

Figura 5. Valoración de la contribución de los usuarios en la producción informativa. Elaboración propia. 


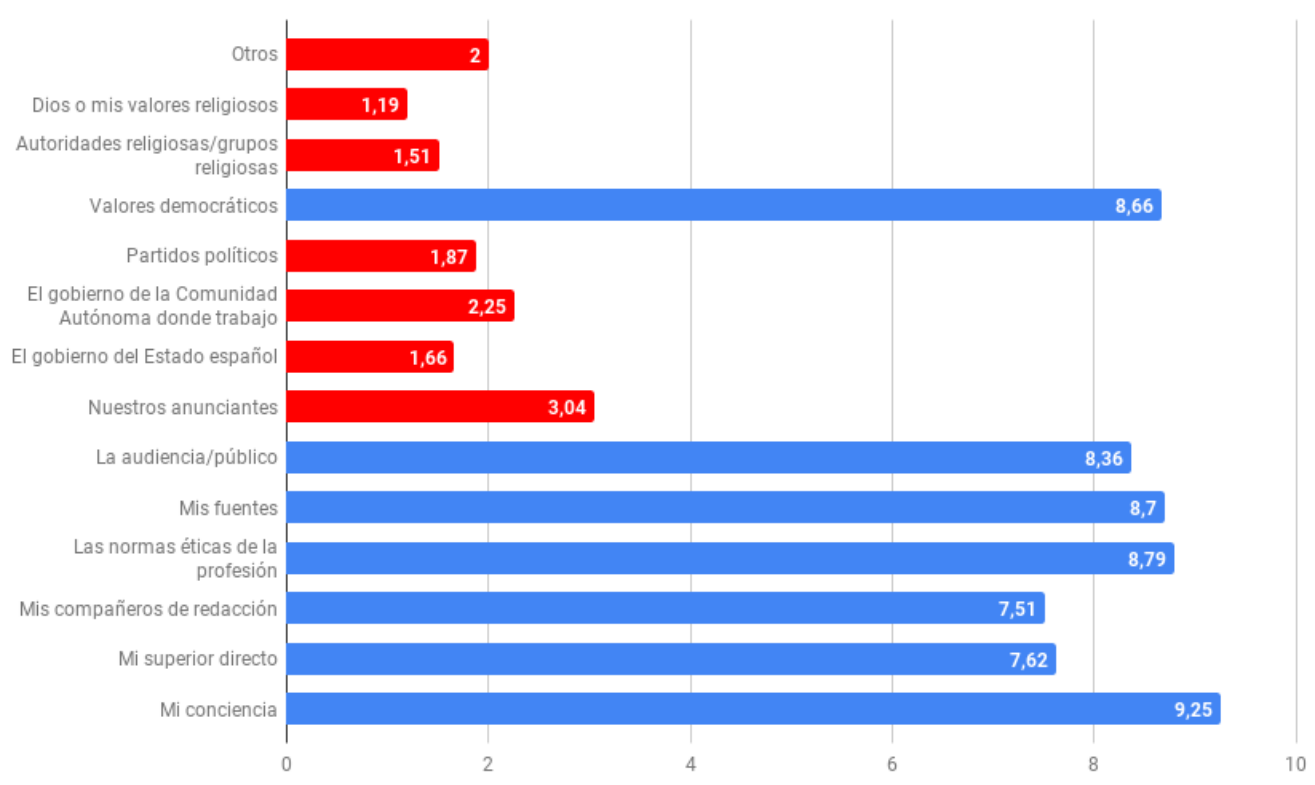

Figura 6. Valoración de la responsabilidad del periodista en el ejercicio de la profesión. Elaboración propia.

\subsection{4. ¿Ante quién se sienten responsables los periodistas catalanes?}

Ante esta pregunta, los periodistas catalanes sitúan con un 9,25 su conciencia (Figura 6). El segundo valor más considerado por los periodistas es la respuesta a las normas éticas de la profesión (8,79), aunque como se ha visto en la Figura 3 , los códigos éticos no recibían más de 6 puntos de valoración. Sin embargo, no se sienten responsables ante los gobiernos español o catalán, ante los partidos políticos o autoridades religiosas. Esta radiografía entronca con la función básica de la accountability desde una óptica normativa: servir a la sociedad, no a los poderes.

\subsection{Valoración por parte de ciudadanos}

\subsubsection{Percepción de los ciudadanos sobre los supuestos que afectan al periodismo}

En la reflexión sobre las limitaciones de los periodistas en su profesión, una de las primeras que aparece en los grupos de discusión se refiere a la necesidad de ganarse la vida en un mundo bastante precarizado y controlado por grandes medios.

Si tú quieres comer, que te contrate alguien, que se compren las noticias, tú te pones tus propias limitaciones. Si quieres hacer una prensa libre y no vives de eso, igual tienes muchísima más capacidad. (GD-M)

El resultado de esta situación repercute en la libertad del periodista, en su libertad para expresar su opinión, para dar la noticia, para hacer autocrítica de su trabajo. También existe un acuerdo generalizado en valorar la profesión de periodista como muy sujeta a condicionantes externos, especialmente respecto a la línea editorial del medio en el que trabaja. La tendencia, por tanto, es a justificar a los periodistas por las presiones que reciben diariamente en cuanto a qué informar, de qué manera, cuándo, qué opiniones expresar y cuáles no.

Yo tengo la obligación moral como persona de hacer mi trabajo correctamente, el periodista también tiene su obligación moral de hacerlo correctamente; ahora bien, ¿es totalmente libre para explicar la historia que quiere en el momento que quiere? No, no es libre. (GD-M)

El factor de la inmediatez, tan importante en la era de la información, acaba anteponiendo la publicación rápida a la noticia reflexionada y contrastada. La demanda de los medios es una noticia corta, que llame la atención y lo más rápido posible. Este hecho se transforma en una limitación relevante para el profesional del periodismo.

Yo es que creo que cada periodista tiene un problema muy gordo, y es la inmediatez de la noticia. Tiene que ser ya, y tiene que ser corta y que llame la atención. (GD-H)

\subsubsection{Percepción de los ciudadanos sobre los instrumentos de accountability}

Para comenzar a tratar el tema de los instrumentos de rendición de cuentas de la profesión para garantizar la ética en los medios se realiza una pregunta abierta y varias personas destacan la necesidad de establecer mecanismos de autorregulación en el periodismo actual. 
Yo creo que tiene un problema y es que no se autorregula, la profesión de periodismo no tiene una autorregulación como la que pueda tener por ejemplo nosotros, los profesionales médicos. En tanto que son profesionales, están obligados a tener una autorregulación y esto no se cumple. Dentro de ellos, cada uno hace lo que le parece sin que luego ello tenga ningún tipo de consecuencia. (GD-M)

A veces oyes, ves, lees, cosas que creo que no están a la altura de lo que debería ser un referente de lenguaje, de comunicación, de respeto. (GD-M)

Las recomendaciones y códigos éticos se valoran de forma positiva e incluso se propone que estos instrumentos sean compartidos por todos los medios.

Para mí el código ético es fundamental y pienso que se debería seguir más en todos los medios. Yo echo en falta lo que es la profesión, al fin y al cabo, donde te enseñan a informar de forma veraz, objetiva y sin ofender a nadie; que realmente son tres cosas que para mí son claves. $(G D-H)$

Pero es que además tendría que ser común a todos los medios, común a todos los periodistas; no según el medio. $(G D-M)$

La participación de la audiencia en la producción de noticias es cada vez más habitual, ello se debe a la facilidad de la población para aportar información grabada en el teléfono móvil, por ejemplo. Casi cualquier testigo hoy en día cuenta con una cámara en mano que rápidamente puede publicar en las redes o enviar a un medio de información. Los ciudadanos ponen diferentes ejemplos de la utilidad de esta contribución por parte de la audiencia.

Cuanta más información tienes de la gente que ha vivido la noticia, mejor. Por eso hay muchas veces que las noticias se basan en la recogida de vídeos o de imágenes del público en general. Ahora es muy fácil grabar un vídeo, mandarlo a la televisión o a un medio y que con eso construyan una noticia y le puedan dar contraste en la medida de lo posible. (GD-M)

Los chats o encuentros digitales con lectores son poco conocidos y utilizados. Aquellos que han participado en alguno reconocen que son interesantes y que son de carácter más lúdico que crítico o reflexivo.

Sí, yo he participado en alguno pues de estos cuando en lugar de entrevistar a un periodista podemos los lectores de un periódico entrevistar a una persona que nos interesa, y creo que está bien porque ya que nos interesa esa personalidad pues a lo mejor se nos ocurren preguntas distintas a las que haría un periodista. (GD-M)

A mí lo de los encuentros digitales con lectores me ha parecido muy interesante, no lo puedes usar con todo el mundo, pero sí que me parece una buena manera de poder llegar. (GD-H)

Por lo que concierne a las entidades o instituciones encargadas de supervisar la ética del periodismo (colegios profesionales, consejos, asociaciones y observatorios), los participantes muestran un desconocimiento general de sus funciones.

Perdona la ignorancia, pero consejos de la información, ¿qué son? ¿Son a nivel estatal, a nivel autonómico? Porque en el momento que lo controle alguien va a estar mediatizado. (GD-M)

Creo que en Cataluña hay el CAC, no sé si es así, voy un poco perdido; pero sí que hecho un poco de menos quizás en prensa nacional que haya un consejo mínimamente regulador (GD-H)

\subsubsection{La contribución ciudadana en el proceso periodístico}

Respecto a la implicación de la audiencia en los procesos de producción, corrección y crítica del proceso periodístico los participantes consideran que es muy poco activa contrastando información, que en realidad sigue fielmente a unos cuantos medios o redes de los cuales se fía y que por tanto acaba creyéndose todo lo que se publica en estas redes de información. La tendencia es leer o escuchar medios afines ideológicamente. Esta falta de miras se critica porque se cree que genera audiencias fácilmente manipulables.

Yo creo que el público en general es muy poco crítico con lo que escucha, con lo cual los medios de comunicación, todos tienen su tendencia y entonces cuentan también con la gente que lee el periódico que le es afín y ve la cadena que le gusta más y... la gente contrasta poco y se lo cree todo (GD-M)

\subsubsection{Según los ciudadanos, ¿ante quién deberían sentirse responsables los periodistas?}

En la última parte de los grupos de discusión se preguntaba ¿ante quién es responsable el o la periodista? Los comentarios en este apartado se refieren a la importancia del derecho a la información veraz, a la responsabilidad para con la audiencia o el público.

Yo creo que el periodista tiene que ser muy consciente de la capacidad de influencia que tiene sobre las personas. (GD-M)

Y ante la sociedad. Ante sí mismo, pero más que la audiencia concretamente ante la sociedad entera, sean 
los que los escuchan concretamente o no. (GD-M)

Se produce una discusión sobre la necesidad de ser fieles a las fuentes o ser fieles con la veracidad de la información.

Yo lo de las fuentes no lo tengo nada claro. Yo lo que creo es que debería ser totalmente libre. (GD-H)

No, porque la veracidad de esta fuente tú también te la tienes que cuestionar y si luego al final ves que lo tienes que tirar por otro lado, pues tienes que tirar por otro lado y no hacer caso... (GD-H)

\section{Conclusiones y discusión}

La situación del periodismo en Cataluña se percibe por profesionales y ciudadanos como negativa. En concreto se cuestiona el hecho que la profesión depende de condicionantes externos que influyen de manera decisiva en la calidad informativa. La precarización de muchos profesionales, la concentración empresarial y, como consecuencia de ello, la falta de libertad de expresión y autocrítica perjudican la salud del periodismo, que se ve fuertemente agravada por la feroz competencia entre los medios por conseguir, a toda costa, más audiencia (lo que lleva a dar respuesta a P1).

De los resultados se colige que los ciudadanos en Cataluña reconocen el hecho que el resultado de esta situación repercute en la libertad del periodista, en su libertad para expresar su opinión, para dar la noticia, para hacer autocrítica de su trabajo. También existe un acuerdo generalizado en valorar la profesión de periodista como muy sujeta a condicionantes externos, especialmente respecto a la línea editorial del medio en el que trabaja. La tendencia, por tanto, es a justificar a los periodistas por las presiones que reciben diariamente en cuanto a qué informar, de qué manera, cuándo, qué opiniones expresar y cuáles no.

En un momento en que ya no son excepción la híper-aceleración de los ciclos informativos, la concentración de medios, la multiplicación de canales de difusión, la polarización de los contenidos, el clickbait o la desinformación (Broersma, 2019; Justel et al., 2018; Masip, Ruiz-Caballero y Suau, 2018; Pickard, 2020; Zelizer, 2018), se generaliza una crisis de valores y la pérdida de vocación de servicio de los medios de comunicación. Si a esta situación se le añade la falta de un modelo de negocio esta idea negativa, hasta pesimista del escenario periodístico catalán actual se pone de manifiesto en el discurso de periodistas y ciudadanos.

Si bien Cataluña se ha considerado un escenario fértil para el desarrollo de iniciativas de accountability, mostrando un amplio espectro de instrumentos de rendición de cuentas impulsados de forma interna y externa a los medios (Alsius, 1999; Alsius, Rodríguez-Martínez y Mauri-Ríos, 2018; Gar-
cía-Avilés, 2019; Ramon-Vegas y Mauri-Ríos, 2020), existe entre profesionales y ciudadanos un panorama generalizado de falta de confianza en los mecanismos internos y externos de rendición de cuentas (con ello, se responde P2). La efectividad de estos mecanismos en el periodismo actual es cuestionada, si bien se ven como necesarios. No se observan opciones óptimas para regular los medios, ni desde organismos públicos, ya que se cree están muy influenciados políticamente.

Los ciudadanos muestran un desconocimiento generalizado sobre estos mecanismos, aunque confirman su necesidad, ya que su existencia se contempla como una seguridad relacionada con el derecho a la información y el contacto visible y accesible para que la audiencia pueda remitir quejas sobre el contenido publicado; también se observa como positiva pues podría ayudar a los profesionales del periodismo a hacer mejor su trabajo y a hacerlos más conscientes de la existencia de la audiencia, a quienes se dirigen. Respecto a las entidades o instituciones que velan por la ética del periodismo (colegios profesionales, consejos, asociaciones y observatorios), los participantes muestran un desconocimiento general de sus funciones. En este sentido, cabe destacar que quizá no se están publicitando de manera suficiente, o que en el terreno de la educación mediática -posible línea de trabajo para mejorar esta situación - aún queda mucho trabajo por hacer.

Se ha mencionado que el entorno digital favorece la aparición de nuevas formas de transparencia, autorregulación y supervisión de la calidad informativa, en que la participación de los usuarios juega un papel decisivo. Los chats o encuentros digitales con lectores son poco conocidos y utilizados por los ciudadanos. Aquellos que han participado en alguno reconocen que son interesantes, en la línea de la idea de que estos nuevos instrumentos comparten la conciencia de que los medios son decisivos en la construcción social del imaginario colectivo (Suárez-Villegas, Rodríguez-Martínez, Mauri-Ríos y López-Meri, 2017).

La participación de la audiencia en la producción de noticias es cada vez más habitual (se responde así P3). Esto se explica debido a la facilidad de la población para aportar información grabada en el teléfono móvil, por ejemplo. En el grupo de discusión se ponen diferentes ejemplos de la utilidad de esta contribución por parte de la audiencia, que del mismo modo que en estudios realizados en otros países (Eberwein, 2019; Engelke, 2019), se considera como positiva. El acceso de corrección de errores genera entre las personas participantes una especial simpatía, aunque es un instrumento también bastante desconocido. Los periodistas, por su parte, consideran que las correcciones que el usuario pueda hacer a las noticias publicadas deben tomarse en cuenta, siempre que se haga a través de la página web del medio, pero el hecho que la audiencia pueda comentar los contenidos a través de las redes sociales no es bien valorado lo que equivale a una falta 
de confianza en el usuario como aportador de información. Existe también el temor de que profesionales de la edición acaben desapareciendo como consecuencia de este trabajo de tipo colaborativo y gratuito, en un contexto de inmediatez que resulta ya desalentador para las figuras de la edición. Estas apreciaciones conectan con el escepticismo sobre ciertos mecanismos de participación mostrado por profesionales de varios países europeos (Fengler et al., 2015). Este hallazgo también sugiere que, en un escenario marcado por la multiplicación de las posibilidades de participación, los periodistas catalanes pueden estar experimentando el fenómeno que Porlezza (2019, p. 2) denomina "fatiga participativa".

Por otro lado, desde el punto de vista de los ciudadanos la posibilidad de implicarse en los procesos de producción, corrección y crítica del proceso periodístico es poco aprovechada, ya que en realidad se sigue fielmente a unos cuantos medios o redes en que se confía y se acaba creyendo todo lo que publican.

Por último, periodistas y ciudadanos participantes creen que el periodista debería sentirse responsable en primer lugar ante la audiencia, el público o incluso a la ciudadanía en general, a las normas éticas de la profesión, a sus fuentes de información, a los valores democráticos (respondiendo así P4). Los profesionales no se sienten responsables ante los gobiernos español o catalán, ante los partidos políticos o autoridades religiosas, y los ciudadanos creen necesario que los periodistas cuestionen sus fuentes, y a la vez muestren un respeto en el tratamiento de la información que no dé pie a malinterpretar o tergiversar esa información. El rechazo por parte de los periodistas catalanes a la sumisión a los poderes políticos, económicos y religiosos es consistente con los resultados obtenidos en investigaciones realizadas en otros países europeos (Harro-Loit, 2015).

A la luz de los resultados obtenidos en el presente estudio, se considera oportuna la formulación de las siguientes propuestas de actuación dirigidas a los medios y a otros actores relevantes en el proceso comunicativo:

- Actuación desde los medios de comunicación: mayor formación y visibilización. A tenor de los hallazgos del estudio, se propone la realización de actividades de formación en las redacciones catalanas sobre los diferentes instrumentos de rendición de cuentas internos y externos, a fin de que los profesionales puedan conocerlos e incorporarlos a su quehacer periodístico. Es clave que los periodistas aprendan de experiencias de éxito impulsadas por otros medios nacionales y en otros contextos periodísticos, a fin de poder experimentar con ellos y valorar la pertinencia de implementarlos o de revisar los instrumentos ya existentes. También se debe apostar por la correcta visibilización de dichos instrumentos para que los usuarios sean conscientes de su existencia. En particular, con el objetivo de fomentar la participación ciudadana, se deben explicar todas las opciones que los usuarios tienen disponibles para contribuir a la producción de noticias, criticar las informaciones publicadas y advertir a las redacciones de los errores cometidos. Como señalan Suárez-Villegas et al. (2020, p. 11), "los medios deben educar a los usuarios en los beneficios de una participación constructiva y que contribuya a aportar puntos de vista que completen y enriquezcan la tarea periodística".

- Actuación desde fuera de los medios de comunicación: fomentar la alfabetización mediática. Varios actores relevantes (colegios y asociaciones profesionales, organizaciones independientes, instituciones educativas) deben apostar por impulsar actividades de alfabetización mediática en formato presencial y virtual. La realización de jornadas, seminarios y talleres puede ayudar a los ciudadanos a comprender los retos y condicionantes del periodismo actual, así como las distintas herramientas que tienen a su alcance para monitorizar la calidad de los medios y contribuir a la mejora del periodismo. Comunicar la existencia de colegios profesionales, asociaciones, observatorios y otros espacios de crítica periodística es fundamental para desarrollar una ciudadanía activa y crítica.

A raíz de los resultados obtenidos, futuras investigaciones deberán monitorizar los patrones de continuidad y cambio en la manera en como los periodistas y ciudadanos perciben la situación del periodismo en Cataluña, la efectividad de los instrumentos de accountability, el papel de los usuarios y la responsabilidad profesional ante varios agentes. Para ello, será necesario seguir aplicando técnicas de investigación cuantitativas y cualitativas como encuestas y grupos de discusión. Complementariamente, la realización de entrevistas en profundidad con miembros de estos colectivos puede contribuir a conocer mejor sus experiencias y puntos de vista (Tracy, 2020). Dichas entrevistas también pueden ayudar a obtener recomendaciones y propuestas que puedan revertir en una mejora de la práctica periodística y el producto resultante.

En paralelo, futuros trabajos deberán radiografiar las páginas web y otros canales digitales de los medios de comunicación catalanes, para comprobar en qué medida estos incorporan instrumentos de accountability tradicionales e innovadores que fomenten la transparencia, la autorregulación y la participación del público. La aplicación del análisis de contenido a los sitios web y perfiles de redes sociales de las organizaciones periodísticas deviene imprescindible para acometer esta tarea. El desarrollo de una propuesta de análisis estandarizada permitirá no sólo examinar la presencia de los instrumentos de accountability en los medios catalanes, sino que facilitará el desarrollo de estudios comparativos que tengan en cuenta medios de varios territorios en España y en otras culturas periodísticas a nivel internacional. 


\section{Nota}

Este artículo pertenece al proyecto de investigación "Accountability y Culturas Periodísticas en España. Impacto y propuesta de buenas prácticas en los medios de comunicación españoles", referencia MINECO/FEDER, UE, ref: CS02015-66404-P, financiado por el Ministerio de Economía y Competitividad y el Fondo Europeo de Desarrollo Regional (FEDER).

\section{Referencias}

Almiron, N., Narberhaus, M., y Mauri-Ríos, M. (2016). Mapping media accountability in stateless nations: the case of Catalonia. Catalan Journal of Communication \& Cultural Studies, 8(2), 207-225. https:// doi.org/10.1386/cjcs.8.2.207_1

Alsius, S. (1999). Codis ètics del periodisme televisiu. Pòrtic.

Alsius, S. (Ed.) (2010). The ethical values of journalists. Field research among media professionals in Catalonia. Generalitat de Catalunya.

Alsius, S., Rodríguez-Martínez, R., y Mauri-Ríos, M. (2018). New formats and old crisis. En T. Eberwein, S. Fengler, y M. Karmasin (Eds.), The European Handbook of Media Accountability (pp. 243-250). Routledge.

Aznar, H. (1999). Ética y periodismo. Paidós.

Aznar, H., y Serrano-Moreno, J. (2017). Autorregulación de la comunicación: análisis de las cien primeras resoluciones de la Comisión de Arbitraje, Quejas y Deontología de la FAPE. El Profesional de la Información, 26(2), 182-191. https://doi.org/10.3145/epi.2017.mar.04

Bertrand, C. J. (2018). Media ethics and accountability systems. Routledge.

Broersma, M. (2019). The legitimacy paradox. Journalism, 20(1), 92-94. https://doi.org/10.1177/1464884918806736

Bryman, A. (2016). Social research methods (5a edición). Oxford University Press.

Campos-Domínguez, E., y Redondo-García, M. (2015). Meta periodismo y transparencia informativa en el periodismo del siglo XXI. Obets. Revista de Ciencias Sociales, 10(1), 185-209. https://doi. org/10.14198/OBETS2015.10.1.07

Chaparro-Domínguez, M.A., Suárez-Villegas, J.C., y Rodríguez-Martínez, R. (2020). Media accountability and journalists: To whom do Spanish professionals feel responsible? Journalism Practice, 14(7), 812-829. https://doi.org/10.1080/17512786.2019.1655470

Christians, C. G., Rotzoll, K. B., y Fackler, M. (1991). Introduction: Ethical foundations and perspectives. En G. Gerbner y M. Siefert (Eds.). Media ethics. Cases and Moral Reasoning (pp. 1-31). Longman.

Craft, S., Vos, T., y Wolfgang, J. D. (2016). Reader comments as press criticism: Implications for the journalistic field. Journalism, 17(6), 677-693. https://doi.org/10.1177/1464884915579332

Díez-Garrido, M., Campos-Domínguez, E., y Calvo, D. (2019). La transparencia de los partidos politicos como estrategia electoral. Una evaluación de sus promesas y sus páginas web. Trípodos, (44), 83-104. https://doi.org/10.51698/tripodos.2019.44p83-104

Eberwein, T., Fengler, S., Lauk, E., y Leppik-Bork, T. (Eds.) (2011). Mapping media accountability - in Europe and beyond. Helbert Von Halem Verlag.
Eberwein, T. (2019). "Trolls" or "warriors of faith"? Differentiating dysfunctional forms of media criticism in online comments. Journal of Information, Communication and Ethics in Society, 1-13. https://doi. org/10.1108/JICES-08-2019-0090

Engelke, K.M. (2019). Enriching the conversation: audience perspectives on the deliberative nature and potential of user comments for news media. Digital Journalism, 8(4), 447-466. https://doi.org/10.108 0/21670811.2019.1680567

Evers, H. (2012). The news ombudsman: Lightning rod or watchdog? Central European Journal of Communication, 5(2), 224-242.

Fengler, S., Eberwein, T., Mazzoleni, G., Porlezza, C., y Russ-Mohl, S. (2014). Journalists and media accountability. An international study of news people in the digital age. Peter Lang Publishing.

Fengler, S. et al. (2015). How effective is media self-regulation? Results from a comparative survey on European journalists. European Journal of Communication, 30(3), 249-266. https://doi. org/10.1177/0267323114561009

Ferruci, P. (2019). The end of ombudsmen? 21st-century journalism and reader representatives. Journalism \& Mass Communication Quarterly, 96(1), 288-307. https://doi.org/10.1177/1077699018805986

García-Avilés, J.A. (1996). Periodismo de calidad. Estándares informativos en la CBS, NBC y ABC. Eunsa.

García-Avilés, J.A. (2019). Examining media accountability in online media and the role of active audiences. En T. Eberwein, S. Fengler, y M. Karmasin (Eds.). Media accountability in the era of post-truth politics: European challenges and perspectives (pp. 270-283). Routledge.

García-De-Torres, E., y Farmer, Y. (2017). Ética en los medios de comunicación: retos y oportunidades para la investigación. El Profesional de la Información, 26(2), 153-157. https://doi.org/10.3145/epi.2017. mar.01

Hafez, K. (2002). Journalism ethics revisited: a comparison of ethics codes in Europe, North Africa, the Middle East, and Muslim Asia. Political Communication, 19(2), 225-250. https://doi. org/10.1080/10584600252907461

Hallin, D.C., y Mancini, P. (2004). Comparing media systems: three models of media and politics. Cambridge University Press.

Hanitzsch, T., Van Dalen, A., y Steindl, N. (2018). Caught in the Nexus: A Comparative and Longitudinal Analysis of Public Trust in the Press. The International Journal of Press/Politics, 23(1), 3-23. https://doi. org/10.1177/1940161217740695

Harro-Loit, H. (2015). Journalists' Views About Accountability to Different Societal Groups. Journal of Media Ethics: Exploring Questions of Media Morality, 30(1), 31-43. https://doi.org/10.1080/089005 23.2014.985296

Heikkilä, H., Domingo, D., Pies, J., Gtowacki, M., Kuś, M., y Baisnée, O. (2012). Media Accountability Goes Online: A Transnational Study on Emerging Practices and Innovations. Journalism Research and Development Centre. http://www.mediaact.eu/fileadmin/user_upload/ WP4_Outcomes/WP4_Report.pdf

Herrera-Damas, S., Maciá-Barber, C., y Luengo-Cruz (2018). When one is not enough. Professional Perception of Traditional Ethical Mechanisms for New Times in Journalism. Estudios sobre el Mensaje Periodístico, 24(1), 213-231. https://doi.org/10.5209/ESMP.59946

Justel, S., Micó, J.L., y Ordeix, E. (2018). Governing the Media: Web Analytics in Spanish Newsrooms. Trípodos, (42), 93-106.

Karlsson, M., Clerwall, C., y Nord, L. (2017). Do not stand corrected. 
Transparency and users' attitudes to inaccurate news and corrections in online journalism. Journalism \& Mass Communication Quarterly, 94(1), 148-167. https://doi.org/10.1177/1077699016654680

Luengo-Cruz, M., Maciá-Barber, C., y Requejo-Alemán, J.L. (2017). Evaluating organisational ethics in Spanish news media. Journalism, 18(9), 1142-1162. https://doi.org/10.1177/1464884916643682

Maciá-Barber, C. (2006). La figura del defensor del lector, del oyente y del telespectador. Universitas.

Masip, P., Ruiz-Caballero, C., y Suau, J. (2018). Monitoring Media Pluralism in Europe: Application of the Media Pluralism Monitor 2017 in the European Union, FYROM, Serbia \& Turkey. Country Report: Spain. The Centre for media pluralism and media freedom. https:// cmpf.eui.eu/wp-content/uploads/2018/11/Spain_MPM2017_country-report.pdf

Masip, P., Ruiz-Caballero, C., y Suau, J. (2019). Active audiences and social discussion on the digital public sphere. Review article. El Profesional de la Información, 28(2), e280204. https://doi.org//10.3145/ epi.2019.mar.04

Mauri-Ríos, M., y Ramon-Vegas, X. (2015). Nuevos sistemas de rendición de cuentas de la información periodística. Una exploración del escenario online español. El profesional de la información, 24(4), 380-389. https://doi.org/10.3145/epi.2015.jul.04

Mauri-Ríos, M., Rodríguez-Martínez, R., Figueras-Maz, M., y Fedele, M. (2018). Press councils as a traditional instrument of media self-regulation: The perceptions of European journalists. Journal of Applied Journalism \& Media Studies, 7(2), 221-243. https://doi. org/10.1386/ajms.7.2.221_1

Moreno-Gil, V. (2019). Accountability en la prensa española: la publicación de los escritos de rectificación. Anàlisi: Quaderns de Comunicació i Cultura, (61), 55-75. https://doi.org/10.5565/rev/analisi.3231

Pastor, L. (2010). Teoría de las cartas al director. La gestión periodística del público. UOC.

Peek, L, y Fothergill, A. (2009). Using focus groups: Lessons from studying daycare centers, 9/11, and Hurricane Katrina. Qualitative Research, 9(1), 31-59. https://doi.org/10.1177/1468794108098029

Pérez-Díaz, P., Zamora-Medina, R., y Arroyas-Langa, E. (2020). Between Self-Regulation and Participatory Monitoring: Comparing Digital News Media Accountability Practices in Spain. Media and Communication, 8(2), 112-123. http://dx.doi.org/10.17645/mac. v8i2.2721

Pickard, V. (2020). Democracy without journalism? Confronting the misinformation society. Oxford University Press.

Porlezza, C. (2019). From participatory culture to participatory fatigue: The problem with the public. Social Media + Society, 1-4. https://doi.org/10.1177/2056305119856684

Raeymaeckers, K. (2005). Letters to the editor: A feedback opportunity turned into a marketing tool. European Journal of Communication, 20(2), 199-221. https://doi.org/10.1177/0267323105052298

Raeijmaekers, D., y Maeseele, P. (2015). Media, pluralism and democracy: what's in a name?. Media, Culture \& Society, 37(7), 1042-1059. https://doi.org/10.1177/0163443715591670

Ramon-Vegas, X., Mauri-Ríos, M., y Alcalá-Anguiano, F. (2016). Transparencia informativa, autorregulación y participación del público. Comunicación y Sociedad, (25), 101-125. https://doi.org/10.32870/cys. v0i25.4423

Ramon-Vegas, X., Billings, A.C., y Rojas-Torrijos, J.L. (2019). Interviews with Former ESPN Ombudsmen / Public Editors Kelly McBride, Robert Lipsyte, and Jim Brady. International Journal of Sport Communication, 12(1), 28-35. https://doi.org/10.1123/ijsc.2018-0127

Ramon-Vegas, X., y Mauri-Ríos, M. (2020). Participación de la audiencia en la rendición de cuentas de los medios de comunicación: instrumentos de accountability y su percepción por parte de los ciudadanos españoles. RAEIC, Revista de la Asociación Española de Investigación de la Comunicación, (7), 50-76. https://doi.org/10.24137/ raeic.7.13.3

Rodríguez-Martínez, R., López-Meri, A., Merino-Arribas, A., y MauriRíos, M. (2017). Instrumentos de rendición de cuentas en España. Análisis comparativo en Cataluña, Galicia, Madrid y Valencia. El Profesional de la Información, 26(2), 255-266. https://doi.org/10.3145/ epi.2017.mar.12

Rodríguez-Martínez, R., Mauri-Ríos, M., y Fedele, M. (2017). Criticism as an accountability instrument: the opinion of Spanish journalists. Communication \& Society, 30(1), 57-72. https://doi. org/10.15581/003.30.1.57-72

Rojas-Torrijos, J.L., y Ramon-Vegas, X. (2017). Accountability en las redes sociales. Libros de estilo en continua evolución y retroalimentación a través de Twitter. Revista Latina de Comunicación Social, 72, 915-941. https://doi.org/10.4185/RLCS-2017-1200

Spiller, R., Degen, M., Kronewald, E., y Guertler, K. (2016). Media watchblogs as an instrument of media accountability: An international survey. Journal of Applied Journalism \& Media Studies, 5(2), 151-176. https://doi.org/10.1386/ajms.5.2.151_1

Suárez-Villegas, J.C. (2015). Self-regulation of offline and online journalism in Spain in the experience of the arbitration, complaints and ethics commission. Communication \& Society, 28(3), 135-149. https:// doi.org/10.15581/003.28.3.135-149

Suárez-Villegas, J.C., Rodríguez-Martínez, R., Mauri-Ríos, M., y LópezMeri, A. (2017). Accountability y culturas periodísticas en España. Impacto y propuesta de buenas prácticas en los medios de comunicación españoles (MediaACES). Revista Latina de Comunicación Social, (72), 321-330. https://doi.org/10.4185/RLCS-2017-1167

Suárez-Villegas, J.C., Rodríguez-Martínez, R., y Ramon-Vegas, X. (2020). Pluralismo informativo en la era de la deliberación digital: percepciones de los periodistas y ciudadanos españoles. El Profesional de la información, 29(5), e290525. https://doi.org/10.3145/ epi.2020.sep.25

Suárez-Villegas, J.C., Zuberogoitia, A., y Gostín, A. (2019). Instrumentos de rendición de cuentas externos a los medios: los casos de Andalucía y la CAPV. Estudios sobre el Mensaje Periodístico, 25(1), 529-546. https://doi.org/10.5209/ESMP.63744

Tracy, S. (2020). Qualitative Research Methods. Wiley-Blackwell.

Valera-Ordaz, L. (2017). Comparing the democratic value of Facebook discussions across the profiles of Spanish political candidates during the 2011 general election. Revista internacional de sociología, 75(1), e052. https://doi.org/10.3989/ris.2017.75.1.15.119

Van Dalen, A., y Deuze, M. (2006). Readers' advocates or newspapers' ambassadors? European Journal of Communication, 21(4), 457-475. https://doi.org/10.1177/0267323106070011

Wahl-Jorgensen, K. (2002). Understanding the Conditions for Public Discourse: four rules for selecting letters to the editor. Journalism Studies, 3(1), 69-81. https://doi.org/10.1080/14616700120107347

Weischenberg, S., Malik, M., y Scholl, A. (2006). Journalismus in Deutschland. Media Perspektiven, (7), 346-361. 
Wimmer, R.D., y Dominick, J.R. (2011). Mass media research: an introduction (9a edición). Cengage Learning.

Zelizer, B. (2018). Epilogue: Timing the study of news temporality. Journalism, 19(1), 111-121. https://doi.org/10.1177/1464884916688964

\section{CV}

Marta Narberhaus Martínez. Profesora de la Facultad de Ciencias de la Comunicación de la Universitat Internacional de Catalunya. Doctora en Comunicación por la Universitat Pompeu Fabra y miembro del grupo de investigación JOVIS.com de la UPF. Su investigación y docencia se centran principalmente en los ámbitos de la educación mediática, la ética periodística y la calidad informativa en medios audiovisuales. Ha participado en proyectos de investigación competitivos nacionales y europeos (Transparencia y Rendimiento de cuentas en la Información Periodística, CS02012-39138; Accountability y Culturas Periodísticas en España. Impacto y propuesta de buenas prácticas en los medios de comunicación españoles, CS02015-66404-P; Integrated Journalism in Europe, 528057-LLP-1-2012-1-ES-FR) y ha sido investigadora visitante en la School of Journalism, Media \& Cultural Studies (Cardiff University, Reino Unido) y en el Institut für Kommunikationswissenschft und Medienforschung (LMU-München, Alemania). https://uic-es.academia.edu/MartaNarberhaus.

Xavier Ramon Vegas. Profesor del Departamento de Comunicación de la Universitat Pompeu Fabra. Doctor en Comunicación por la UPF. Miembro del Grup de Recerca en Comunicación Política, Periodismo y Democracia (POLCOM-GRP). Su investigación y docencia se centran en los ámbitos de la ética periodística, la rendición de cuentas de los medios y la comunicación deportiva. Ha publicado en revistas académicas como Digital Journalism, Journalism Studies, El Profesional de la Informa- ción, Revista Latina de Comunicación Social, Communication \& Sport, Ethical Space, Estudios sobre el Mensaje Periodístico o Revista de Comunicación. Ha realizado estancias en la University of Stirling y la University of Glasgow (Reino Unido), la University of Alabama (Estados Unidos) y el Centro de Estudios Olímpicos del Comité Olímpico Internacional (Suiza). Ha participado en tres proyectos competitivos I+D financiados por el Gobierno de España sobre rendición de cuentas de los medios de comunicación y plataformas de fact-checking. https://upf. academia.edu/XavierRamon.

Cristina Perales-García. Profesora de periodismo en la Universitat Pompeu Fabra. Doctora en Periodismo y Ciencias de la Comunicación con Mención Europea por la Universitat Autònoma de Barcelona; posgrado en Mediación y Resolución de Conflictos por la UOC y posgrado en reporterismo ENG, por la UPF. Actualmente es coordinadora docente del Grado en Periodismo de la UPF. Miembro del comité de evaluación de la European Commission (Research Executive Agency, REA) de proyectos de investigación en comunicación en la convocatoria pública de los proyectos H2020 Marie Skłodowska-Curie Individual Fellowships. Miembro del Grupo de Investigación en Comunicación Política, Periodismo y Democracia (POLCOM-GRP). Está especializada en el campo del periodismo y la comunicación política, así como en el estudio de los discursos periodísticos y los estudios de género en el periodismo. Ha realizado contribuciones científicas en revistas y en libros sobre la responsabilidad de los medios en la representación de los conflictos nacionales. https://www.upf.edu/web/cristina-perales.

\section{осм Observatorio de Cibermedios}

https://observatoriocibermedios.upf.edu/

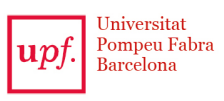

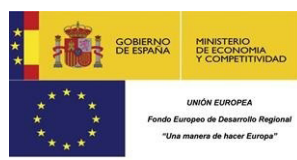

El Observatorio de Cibermedios es una producción del Grupo de Investigación en Documentación Digital y Comunicación Interactiva (DigiDoc) del Departamento de Comunicación de la Universitat Pompeu Fabra.

El Observatorio de Cibermedios (OCM) forma parte del proyecto del Plan Nacional "Narración interactiva y visibilidad digital en el documental interactivo y el periodismo estructurado". RTI2018-095714-B-C21 (MINECO/FEDER), Ministerio de Ciencia, Innovación y Universidades (España). 